\title{
Thermodynamical Significance of Salinities in Seawater and Human Blood and the Effect of Dissolved Oxygen \\ A Review
}

\author{
Shizuo FujIwara \\ The Research Institute for Information and Knowledge, Kanagawa University, \\ Rokkakubashi, Yokohama 221
}

\begin{abstract}
Solute entities in solution are assumed to take one of two phases depending on the concentration: one can be seen in the lower concentration range, where each of the solute entities is dispersed in the media of the solution, and the other in the higher range, where the solutes interact with each other. Spectroscopic and thermal investigations and quantum chemical calculations have revealed the presence of a critical concentration which can be taken as the interface of these two phases. An enormously enhanced fluctuation of the refractive index is observed in solutions at the critical concentration. Furthermore, such fluctuations disappear when oxygen is removed from the solution. The values of the critical concentration of the aqueous sodium chloride solutions coincide with the salinities of the seawater and the human blood. These findings are discussed in connection with the laws of thermodynamics.
\end{abstract}

Keywords Thermodynamics law, salinity, seawater, human blood, oxygen effect

1 Introduction

2 Critical concentration for binary component solution

2.1 Critical concentration for propylene carbonate-water mixtures

2.2 ESR of aqueous solution of copper(II) ions

2.3 Heats of infinite dilution of aqueous sodium chloride solutions

2.4 Quantum chemical calculation on solvation number
3 Fluctuation of refractive index in solution of seawater salinity

4 Fluctuation of refractive index in solution of human blood salinity

5 Thermodynamical significance of salinities of seawater and human blood and their relation with dissolved oxygen

6 Significance of the salinities of seawater and human blood in nature: Conclusive remark

\section{Introduction}

Electrolyte solutions, especially the aqueous sodium chloride solutions, play essential roles in the chemical processes in nature. Therefore, extensive efforts have been made for a long time for the elucidation of the structure of such solutions or for the determination of the entities that make up the properties of these solutions.

More recently, spectroscopic investigations as well as quantum chemical calculations have led to the conclusion that the solutions may be classified into two phases, one in which each solute behaves as an individual independent of the others, and the other where all solutes behave as a united body with mutual interaction. This conclusion has stimulated further investigation on the thermodynamical basis of the problem. Some elucidation is needed on the significance of the salt concentrations of the seawater and the human blood as well as on the role of the dissolved oxygen.

The first law of thermodynamics refers to the conservation of the energy which is held by a chemical system, and the second to its constitution as it is formed of two phases, of entropy and inner energy. The entropy and the inner energy are convertible to each other within the system when the system is in equilibrium with the surroundings.

It is essential that those two laws can hold only in the case where the system is in equilibrium. In other words, the laws of thermodynamics do not tell much about a system composed of two subsystems which are not in equilibrium to each other or to the outside. Then a question arises on the energy flow between those two subsystems, whether they keep themselves thermally inert or not.

Spectroscopic investigations on the structural change of the aqueous electrolyte solutions suggest the presence of a critical concentration at which the solution 
behaves as an interface of two subsystems as cited above. The magnitude of that critical concentration varies depending on the species of the electrolytes as well as on the mechanism of the interface. The present article will describe first what evidence the experiments and quantum chemical calculation offer for the presence of a critical concentration, i.e., that of the interface, and then the thermodynamical behavior of the solution of the specific concentration. It will be shown also that the oxygen dissolved in the solution plays a significant role in the flow of the thermal energy of the system.

\section{Critical concentration for binary component solution}

Investigations on the structures of the aqueous electrolyte solutions have been made by various methods, such as the X-ray spectroscopy, NMR, particularly proton NMR and C-13 NMR, UV, and IR. They have mainly concerned with the structures of the solutes and the solute-solvent interactions, such as the solvation. Namely, they have been more focussed on the microstructures of the solution and not much on the bulk nature of the solution. Thus present article will present some discussion on the latter problem.

\subsection{Critical concentration for propylene carbonate- water mixtures}

Before going into a detailed discussion on the aqueous solution of electrolytic salt, some results of the spectroscopic investigation on a simple system of a mixture of propylene carbonate (to be abbreviated as PC hereafter) and water, will be shown first. ${ }^{1}$

Propylene carbonate has relatively inert groups of $-\mathrm{CH}_{3}$ and $>\mathrm{CH}_{2}$ and fairly waterphilic groups of $>\mathrm{CH}$ and $>\mathrm{C}=\mathrm{O}$. As the UV absorption is measured for the mixtures of various concentrations of $\mathrm{H}_{2} \mathrm{O}$ in $\mathrm{PC}$, the spectra clearly refer to the presence of the isosbestic point at around $250 \mathrm{~nm}$ and suggests that the mixtures can be classified into two phases with $0.5 \mathrm{~mol} \mathrm{H}_{2} \mathrm{O} / \mathrm{mol}$ $P C$ as the critical concentration.

A more detailed inside view of the mixtures can be obtained by C-13 NMR. The chemical shifts of C13 NMR in Fig. 1 show that in the solutions of low

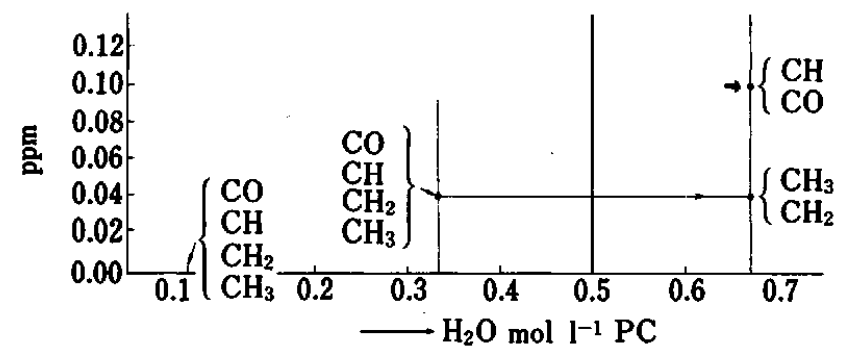

Fig. 1 C-13 NMR shifts (ppm) for mixtures of propylene carbonate and water. The abscissa refers to the concentration of water added to propylene carbonate and the ordinate to the shift of each function from the value at $\mathrm{H}_{2} \mathrm{O}=0$. concentration of $\mathrm{H}_{2} \mathrm{O}, 0.01$ or $0.067 \mathrm{~mol} \mathrm{H}_{2} \mathrm{O} / \mathrm{mol} \mathrm{PC}$, the resonant positions of $\mathrm{C}-13 \mathrm{NMR}$ for all functional groups are the same as those of the neat PC, whereas, in the mixture of $0.33 \mathrm{~mol} \mathrm{H}_{2} \mathrm{O} / \mathrm{mol} \mathrm{PC}$, the resonant positions of all groups are shifted uniformly by the same amount whithout any specific shifts among each other. However, in the mixture of $0.66 \mathrm{~mol} \mathrm{H}_{2} \mathrm{O}$ / mol PC, the radicals of $>\mathrm{CH}_{2}$ and $-\mathrm{CH}_{3}$ still remain in the same positions as in the solution of 0.33 , but those of $\mathrm{C}-\mathrm{H}$ and $>\mathrm{C}=\mathrm{O}$ are equally shifted from the former positions of the solution of $0.33 \mathrm{~mol} \mathrm{H}_{2} \mathrm{O} / \mathrm{mol} \mathrm{PC}$. These results suggest that the PC molecules in the solutions of lower concentration water behave uniformly, i.e., making no different interaction with water among the functional groups, and accordingly producing no function-dependent chemical shift on carbons. But in the higher concentration range, PC molecules interact with the water molecules specifically, resulting in chemical shifts among different groups. Accordingly, one can assume the presence of a critical concentration in the $\mathrm{PC}-\mathrm{H}_{2} \mathrm{O}$ system. Taking the results of the $\mathrm{UV}$ measurements into consideration, $0.5 \mathrm{~mol} \mathrm{H} \mathrm{H}_{2} \mathrm{O} / \mathrm{mol} \mathrm{PC}$ is referred to as the critical concentration for the system of $\mathrm{H}_{2} \mathrm{O}-\mathrm{PC}$. The point of the latter finding is that the structures of the mixed solutions can be divided into two phases, one where the solute molecules do not form any compound with the solvent and remain independent of each other, i.e., the solute molecules are simply dispersed in the media of the solution, and the other where the solute molecules form compounds with the solvent: the solvated solutes.

\subsection{ESR of aqueous solution of copper(II) ions}

The critical concentration as cited above can be identified in the aqueous solutions of electrolytes. The ESR spectra of cupric ions in aqueous solution is a single line; its line width shows the concentration dependence as shown in Fig. 2. ${ }^{2}$ The line width in the lower concentration range does not show any concentration dependence nor dependence on the species of anions. Also, because the line width is the same in normal water and in heavy water, it has been assumed to be viscosity independent. All these observations have been taken as suggesting that, for low concentrations below $c a .0 .1 \mathrm{~mol} \mathrm{Cu}^{2+} \mathrm{l}^{-1}$, the observed line width

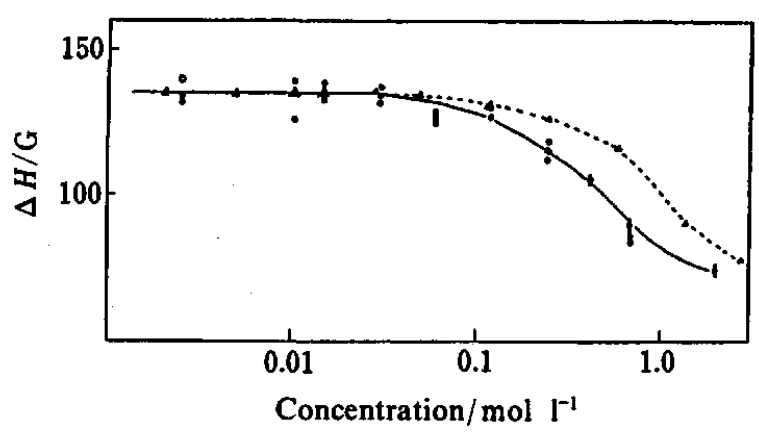

Fig. 2 Concentration dependence of line widths of ESR of $\mathrm{Cu}^{2+}$; ions in aqueous solutions: $O$, sulfate; $\Delta$, nitrate. 
of $\mathrm{Cu}^{2+}$ is determined by the spin-orbit coupling of the cupric ion itself, i.e., by the intrinsic nature of the ions, and not by ion-ion interactions. But the line width in the higher concentration range is concentration and anion dependent. The latter results have been taken as due to the ion-ion interactions which result in the electron exchange narrowing of the widths.

Accordingly, again here, the cupric sulfate or the nitrate solutions refer to $0.1 \mathrm{~mol} \mathrm{Cu}^{2+} \mathrm{l}^{-1}$ as the critical concentration which divides the states of the solution into two phases, one in which the ions are simply dispersed in the media of the solution, and the other in which the ions interact with each other.

\subsection{Heats of infinite dilution of aqueous sodium chloride solutions}

The data of the heats of infinite dilution of the solutions of sodium chloride also present a clear cut critical concentration. ${ }^{3}$ Figure 3 reproduces the data of the concentration dependence of the heats of infinite dilution $\Delta H_{\text {inf.dil. }}$ for the aqueous sodium chloride solutions. The data in Fig. 3 suggest that the solutions can be divided into two phases. The data for the solutions of the concentration lower than ca. $0.5 \mathrm{~mol} \mathrm{l}^{-1}$ of $\mathrm{NaCl}, \Delta H_{\text {inf.dil.'s }}$ are positive and concentration independent, whereas those in the higher are negative and concentration dependent. The fact that the $\Delta H_{\text {inf.dil. }}$ values are positive and concentration independent may be interpreted in terms of the fact that the ions are dispersed in the media of the solution, being independent from each other; this results in a positive

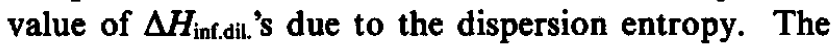

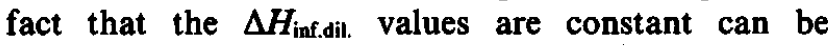
interpreted in the same way. The fact that the values in the higher concentration range are negative and concentration dependent can be interpreted as being due to the ion-ion interactions.

\subsection{Quantum chemical calculation on solvation num- ber}

Experimental studies by various spectroscopic methods $^{4}$ such as X-ray, NMR, or neutron diffraction have led to more knowledge about solutions particularly with respect to the solvation number evaluation for the ions. However, they also led to new questions, due to

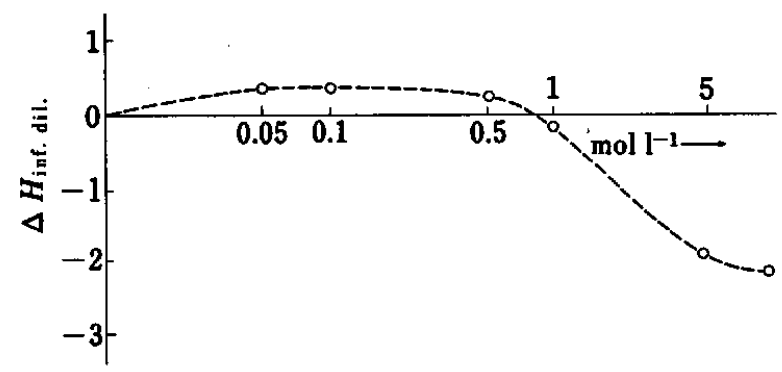

Fig. 3 Heats of infinite dilution of aqueous sodium chloride solutions. problems inherent in these experimental techniques.

Especially, solvation (=solvent coordination) numbers for ions depend strongly on the methods being used for their determination (e.g., vapor pressure measurements, conductivity methods and NMR techniques) and have to be based, in addition, on an arbitrary separation of anionic and cationic contributions to the overall-effect being measured. X-ray diffraction data seem to yield the most well-defined results $^{5}$ in this field, but the results are confined to the first coordination shell. Furthermore, the limitations of the capability of this method can not be denied with respect to the complexities of the system being investigated and the impact of energy exerted during the measurement by the X-rays, in comparison with the interaction energies determining the formation of structural units in solutions.

Theoretical approaches based on quantum chemically evaluated $a b$-initio interaction potentials and their use in Monte Carlo molecular dynamics simulations of solutions have made a new powerful tool available for this field of research. They allow us not only to evaluate solvation numbers and solute-solvent distances from computed radical distribution functions, but also give access to data not available from any experimental technique so far, as for example an analysis of the percent contribution of various coordination numbers in the average solvation number of the ion or solvent molecules.

Thus, a series of calculations has been performed with the focus on Monte Carlo simulations of aqueous
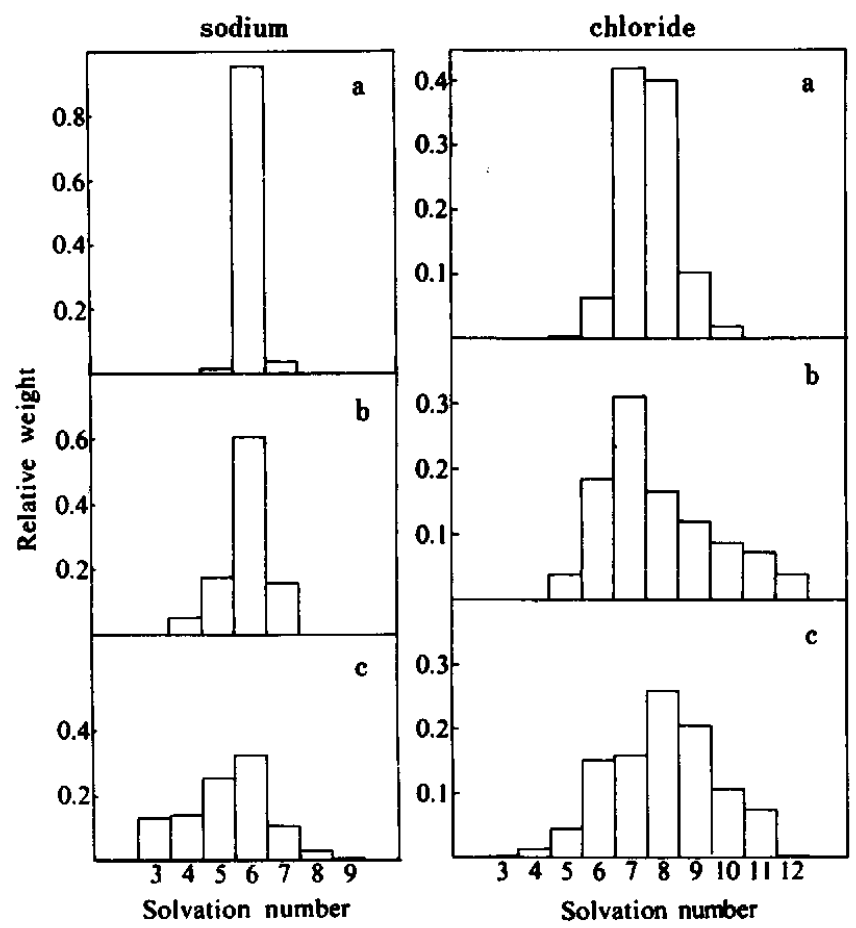

Fig. 4 Distribution of coordination numbers for sodium and chloride ions: $a$, infinite dilution; $b, 0.55 \mathrm{~mol} \mathrm{l}^{-1}$ and $c$, $3.5 \mathrm{~mol} \mathrm{l}^{-1}$. 
Table 1 Solvation numbers from radial distribution functions for $\mathrm{Na} \cdots \mathrm{O}, \mathrm{Cl} \cdots \mathrm{O}$ and $\mathrm{O} \cdots \mathrm{O}$ distances at various concentrations

\begin{tabular}{ccccccc}
\hline \multirow{2}{*}{ System } & \multicolumn{2}{c}{$\mathrm{Na}^{+}$} & \multicolumn{2}{c}{$\mathrm{Cl}^{-}$} & \multicolumn{2}{c}{$\mathrm{H}_{2} \mathrm{O}$} \\
& 1st shell & 2nd shell & 1st shell & 2nd shell & 1st shell & 2nd shell \\
\hline Infinite dilution & 6.0 & - & 7.4 & - & - & - \\
0.55 & 6.1 & 17 & 7.1 & 0 & 5.5 & 20.2 \\
0.35 & 5.4 & 0 & 11.8 & 0 & 10.0 & 0 \\
\hline
\end{tabular}

sodium chloride solution to find the distribution of coordination numbers and the angular distribution in the first solvation shell of the ions. ${ }^{6}$

Table 1 gives the first and second shell solvation numbers of ions and solvent molecules at different concentrations. Figure 4 shows the distribution of the coordination numbers for $\mathrm{Na}^{+}$and $\mathrm{Cl}^{-}$. Data of Table 1 suggest that a fally solvated sodium ion requires 23 water molecules, a fully solvated chloride ion 7-8 water molecules and that "complete" bulk water units consist of a minimum amount of approximately 26 solvent molecules.

On the basis of those data, it has been calculated that in $0.5 \mathrm{~mol} \mathrm{l}^{-1}$ solution about half of the solvent available is used up for ion solvation. In this sense, lower concentrations can be considered as "bulk solvent dominated", whereas concentrations of more than $0.5 \mathrm{~mol} \mathrm{l}^{-1}$ sodium chloride have to be rather designated as "solvation shell water dominated". This peculiality of $0.5 \mathrm{~mol} \mathrm{l}^{-1}$ sodium chloride solution is assumed to refer to the critical concentration cited above. Figure 4 illustrates how the average first shell solvation numbers of sodium are made up statistically of varying concentrations. It can be seen that, with increasing salt concentration, the variation of coordination numbers becomes successively larger, indicating not only a wider variability of structural units, but also a "sharing" of solvation shell waters by more than one ion.

The results of the quantum chemical calculation also suggested that $0.5 \mathrm{~mol} \mathrm{l}^{-1}$ solution is the start of the ion-pair formation. The point of this calculation is that from around $0.5 \mathrm{~mol}^{-1}$ ion pairs can be formed, where the solvent molecules are not separated but rather correspond to the formation of ion-pairs, the socalled "contact ion pairs". This develops a rather concise picture of the structural units of highly concentrated sodium chloride solution as containing almost no independent solvated ions, but rather such contact ion pairs, imbedded in a network of other ion pairs at nearly the statistical distance, and water molecules, whose orientation is influenced more by the sodium ions than by the chloride ions.

The quantum chemical calculation suggests the same conclusion as that obtained before by the experiments, that from above $0.5 \mathrm{~mol} \mathrm{l}^{-1}$ concentration, the composition of the solution is characterized by the formation of ion pairs which are not solvent separated, but rather correspond to so-called "contact ion pairs", and that a larger structure unit containing another three counter ions is formed with a radius of $6.4 \AA$.

\section{Fluctuation of refractive index in solution of seawater salinity}

Analysis of dynamical properties of a chemical system is a problem of essential importance in chemistry. The static figures of molecules or solid materials have been studied fairly well by various experimental methods. However, the dynamic ones, particularly those in solution, remain mostly unknown.

In view of the results referred to above, the aqueous solution of sodium chloride at the concentration of $0.5 \mathrm{moll}^{-1}$ may be taken as the interface of two thermodynamically different phases, one where the dispersed entities of ions dominate and the other where the solvated ions interact with each other and they behave as an ensemble.

The present author has been interested to see the energy flow in and out of the solution of the critical concentration and, accordingly, to measure the fluctuation of the refractive index of the solution. The idea of the latter measurement is that some thermal instability may be present in the specific solution of the critical concentration and the instability may be reflected in the fluctuation of the refractive index of the solution, because the density of the solution is assumed to be directly related with the thermal agitation of a system.

Fluctuations are usually taken as disturbing elements in an experiment, and efforts are made to keep them as small as possible. However, it is also being recognized recently that noise is a source of valuable information. Fluctuations or noise are being investigated in various fields, such as chemistry, physics and biochemistry. Such work is particularly useful for the measurement of properties of any system which are easily perturbed by the measurement. The solution of the critical concentration is assumed to be such a system of interest from the viewpoint of fluctuations, because that solution may present a peculiar condition of an interface of two phases as cited above. In fact, measurements of the fluctuation of refractive indices of aqueous solutions has proven useful.

The refractive index has been measured on the aqueous strong electrolytes, then with focusing on the solutions of the concentrations around $0.5 \mathrm{~mol}^{-1}$ of $\mathrm{NaCl}$ by a normal meter, where a beam of tungsten light goes through the solution and is compared with 


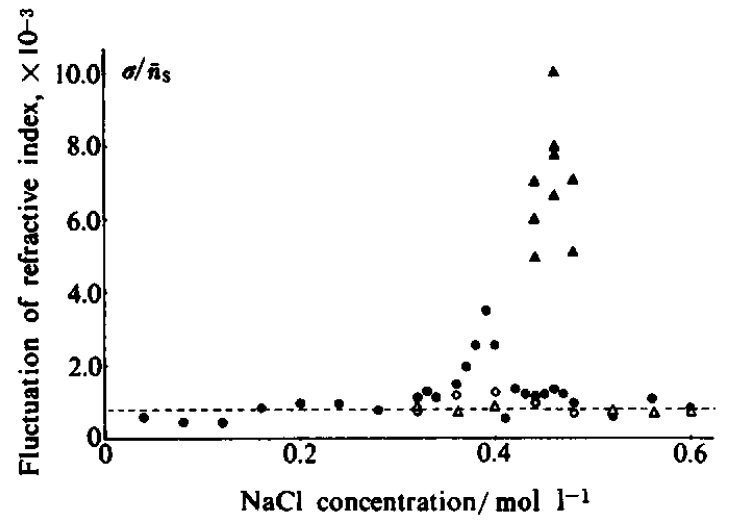

Fig. 5 Fluctuation of refractive index in aqueous sodium chloride solutions, with oxygen (solid) and without oxygen (open) for data $\Delta, \Delta 20 \mathrm{~s}$ and $O, O 1$ s sampling, respectively.

the light passed through the reference sample. ${ }^{7,8}$ The output of the meter is the difference between the refractive indices of two solutions, $\Delta n=n_{\mathrm{s}}-n_{\mathrm{r}}$, where $n_{\mathrm{s}}$ and $n_{\mathrm{r}}$ are the indices of the solution and of the references, respectively. The $\Delta n$ value is presented in four digits. The measurements have been made at 10 , 25 and $35^{\circ} \mathrm{C}$, but mostly at $35^{\circ} \mathrm{C}$. The temperature of the measurements is assumed to have an ambiguity of $\pm 0.1^{\circ} \mathrm{C}$ at the set temperature. The values of $\Delta n$ are sampled by a NEC PC-8801 microcomputer at certain intervals, 0.1, 1, 5 and $20 \mathrm{~s}$. For the experiment on the solutions of around $0.1 \mathrm{moll}^{-1}$ of $\mathrm{NaCl}$ concentration, the data sampling was done by an NEC PC-9801 at an interval of $1 \mathrm{~ms}$.

Figure 5 shows the data of fluctuations, where $\sigma / \bar{n}_{\mathrm{s}}$ in the ordinate refer to the standard deviation of the measurements: i.e., $\sigma$ is the deviation of $\Delta n$ and $\bar{n}_{\mathrm{s}}$ is the average of the refractive index of the sample solution. The data in the figure refer to the results of two different sampling times, of 1 and $20 \mathrm{~s}$. Although some difference is observed in the value of the $\sigma / \bar{n}_{\mathrm{s}}$ peak concentration, it is obvious in the figure that the fluctuation is strongly enhanced in the solutions in the region between 0.4 and $0.5 \mathrm{~mol} \mathrm{l}^{-1}$ of NaCl. The results of Fig. 5 indicate that an anomalously enhanced fluctuation is observed in the refractive index of the solution with the concentration in the range of the critical concentration of the interface. Similar results of the enhancement of the refractive indices at specific concentrations have been noticed with other aqueous alkali halide solutions.?

Similar enhancements have also been evidenced with mixtures of the normal and heavy water as well as with the solution of sodium chloride with the concentration of $0.1 \mathrm{~mol} \mathrm{l}^{-1}$.

Figure 5 also shows the effect of oxygen. The solid symbols in the figure refer to the data for the solutions which carry oxygen, the open ones to those without oxygen. Oxygen was removed in the latter samples simply by introducing nitrogen into the reservoir of the sample solution. Hence, the presence of a small

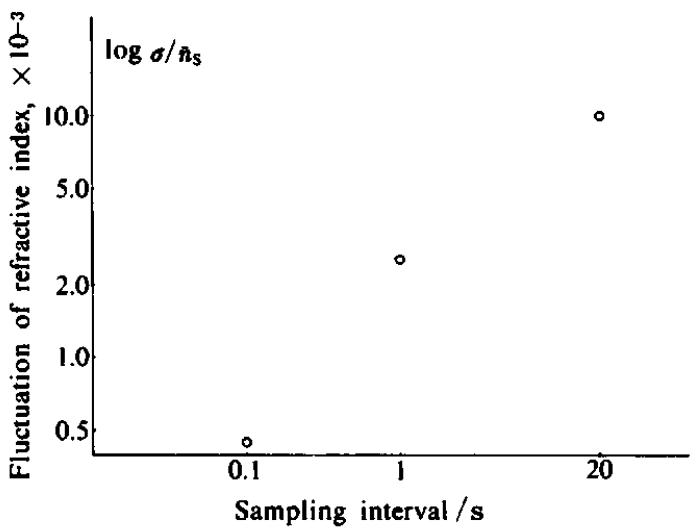

Fig. 6 Logarithmic relation of enhanced fluctuation with the interval of sampling time.

amount of oxygen in the solution cannot be denied. Even so, however, it is obvious from the figure that the enhanced fluctuation which has been observed in the oxygen-carrying solutions disappears when oxygen is removed. This effect of oxygen is assumed to play an important role in the function of the seawater whose salt concentration coincides with the value of the critical concentration of the solvation of ions cited above and in the anomalous fluctuation of the refractive index.

Figure 6 shows the observed results on the relation of the peak height of the enhanced fluctuation with the interval of the sampling time, apparently a logarithmic relation. The result shown in Fig. 6 awaits further detailed investigation.

\section{Fluctuation of refractive index in solution of human blood salinity}

Fluctuation of the refractive index has been measured in aqueous sodium chloride solutions of around $0.1 \mathrm{~mol} \mathrm{l}^{-1}$ concentration, which is the salinity of the human blood. The aim of the measurements is to see if fluctuations of the refractive index can be observed in the human blood salinity solution, which is the other important saline solution in nature beside seawater. Because the solution of the seawater salinity has been evidenced to be an interface of two phases, one dominated by the dispersion entropy of the ions and the other by the inner energy of the mutually interacting solvated ions, it has been assumed that if similar fluctuation were observed with the solution of the salinity of $0.1 \mathrm{~mol} \mathrm{l}^{-1}$ of $\mathrm{NaCl}$, the result could be taken as the evidence for the fact that $0.1 \mathrm{~mol} \mathrm{l}^{-1}$ is another interface of two different phases. In fact, the fluctuation has been observed and the specific concentration of $0.1 \mathrm{moll}^{-1}$ has been interpreted in terms of the break of hydrogen bonding. 9

Before going to the experiment on $0.1 \mathrm{~mol} \mathrm{1}^{-1}$ of $\mathrm{NaCl}$ solution, measurements were made on the mixtures of the normal and the heavy waters in order 
to see the perturbing effect of the heavy water on the normal water. The idea of the latter approach is as follows. The electrolytic ions in $0.1 \mathrm{~mol} \mathrm{l}^{-1}$ of $\mathrm{NaCl}$ aqueous solution may be taken as network breakers for the three dimensional network of the hydrogen bonding. In order to see the effect of heavy water as the most simple case of the breaker, the heavy water has been introduced into the normal water. Measurements of the fluctuation of the refractive index have been done in a way similar to that of the former experiment on the solutions of the seawater equivalent salinity except for the way of sampling of data.

In this experiment, the output of the refractive index meter was directly sampled and processed by a personal computer, PC-9801, of Nippon Electric Company. The output of the meter, which actually refers to a difference in refractive indices of the sample and the reference solutions, is sampled for $1 \mathrm{~min}$ at an intervals of $1 \mathrm{~ms}$. Six thousand data are thus obtained with one measurement run. They are then broken into groups of data of different sampling intervals. Group No. 1 refers to the data with an interval of $1 \mathrm{~ms}$, No. 2 for those of interval $2 \mathrm{~ms}$, No. 3 of $3 \mathrm{~ms}$, and so on. Statistical analysis of the data for each of the groups was carried out by the computer. The fluctuation, $\sigma$, has also been calculated for each group in the same way as done in the former investigation.

The results of measurements for $\mathrm{D}_{2} \mathrm{O} / \mathrm{H}_{2} \mathrm{O}$ are shown in Fig. 7, where the concentration of $\mathrm{D}_{2} \mathrm{O}$ in $\%$ by volume is taken along the $x$ axis, the sampling time interval, i.e., the number of the grouping of data, along $y$ and the fluctuation of the refractive index, $\sigma$, along $z$. The figure presents a line-up of $\sigma$ 's of all groups of data. The front-most line in the figure refers to the fluctuation of group No. 1 for the data of $1 \mathrm{~ms}$ sampling interval, the next line to that of group No. 2 and so on.

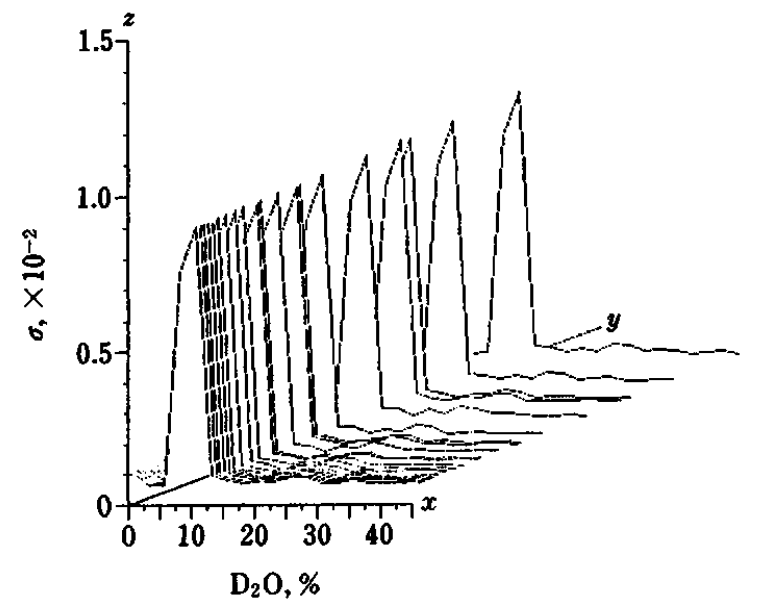

Fig. 7 Fluctuation of refractive index, $\bar{\sigma}$ of $\mathrm{D}_{2} \mathrm{O}-\mathrm{H}_{2} \mathrm{O}$ mixtures. $x$, concentration of $\mathrm{D}_{2} \mathrm{O}(\%), y$, interval of data sampling in $\mathrm{ms}, z$, fluctuation. The front-most line of the data refers to $\bar{\sigma}$ for $1 \mathrm{~ms}$ of $y$, the next line for $\mathbf{2} \mathrm{ms}$, and so on.
It is obvious in the figure that all lines show a peak at the concentration of $15 \%$ and fall to the ground level at $20 \%$. Figure 8 presents the results of the sodium chloride solutions which exhibit a drastic drop of $\sigma$ at the concentration range from 0.03 to $0.10 \mathrm{~mol}^{-1}$ of $\mathrm{NaCl}$ getting almost zero value at the average for the higher concentration range. Again, the $x$ axis in Fig. 8 refers to the concentration of salt, $y$ to the sampling time interval and $z$ to the fluctuation, $\sigma$.

The results of Fig. 7 on the $\mathrm{D}_{2} \mathrm{O} / \mathrm{H}_{2} \mathrm{O}$ system suggest that an anomalously heavy fluctuation of the refractive index occurs in the system of $15-20 \% \mathrm{D}_{2} \mathrm{O}$, which may imply the formula of a system of $\mathrm{D}_{2} \mathrm{O}\left(\mathrm{H}_{2} \mathrm{O}\right)_{4}$.

Actually, a quantum chemical calculation ${ }^{10}$ has shown recently that the tetrahedral coordination is the most probable arrangement of the cluster of water. The tetrahedral structure of hydration has also been presented as the most probable structure for sodium ion. ${ }^{11}$ The five-membered cluster model of water shown in Fig. 9 has been referred to earlier as the most plausible form for the interpretation of the Raman

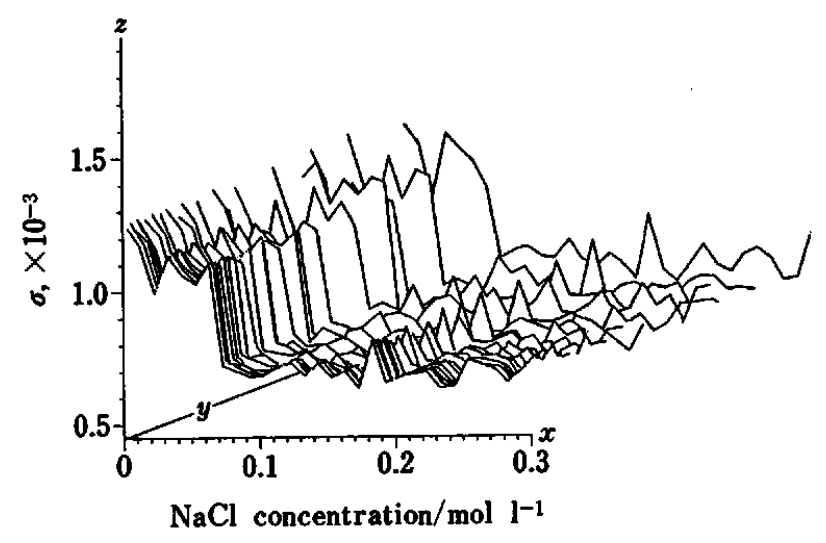

Fig. 8 Fluctuation of refractive index, $\bar{\sigma}$, of aqueous sodium chloride solution. $x$, concentration, $\mathrm{mol} \mathrm{l}^{-1}$ of $\mathrm{NaCl}, y$, interval of data sampling in $\mathrm{ms}$, and $z$, fluctuation.

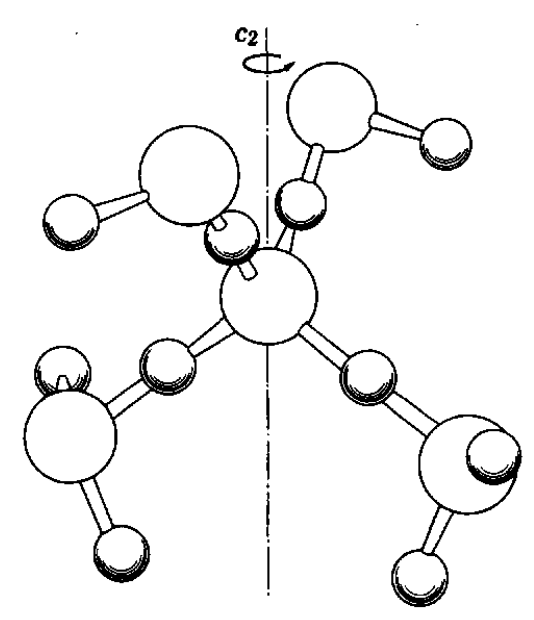

Fig. 9 Five-membered cluster model of water (after Franks ${ }^{2}$ ). 
spectra. ${ }^{12}$

These facts suggest a possible interpretation of the fluctuation of the aqueous sodium chloride solutions with concentrations of around $0.1 \mathrm{~mol}^{-1}$. It is normal that electrolytic ions break the hydrogen bonding of water. This will result in the formation of a cluster of water molecules ${ }^{10}$ or of ions tetrahedrally coordinated by four water molecules."

Then, with the analogy to the case of the solution of $0.5 \mathrm{~mol} \mathrm{l}^{-1}$ of $\mathrm{NaCl}$, the specific concentration of $0.1 \mathrm{~mol} \mathrm{l}^{-1}$ of $\mathrm{NaCl}$ may be taken as what is referred to as the critical concentration for the two competitive phases, one that of the three-dimensional network of hydrogen bonding and the other that of the assembly of the clusters of the water molecules or tetrahedrally solvated ions. The models cited above suggest that the former phase refers to the case where the whole system is the one united and the latter to the assembly of the individual clusters. The former phase may be assumed to be dominated by the inner energy and the latter by the entropy; hence, the specific concentration of $0.1 \mathrm{~mol} \mathrm{1}^{-1}$ of $\mathrm{NaCl}$, which is the salinity of the human blood, is the interface of the two thermodynamically different solutions.

It is furthermore to be noted that the anomalous fluctuations shown in Fig. 7 and 8 have disappeared when the dissolved oxygen is removed: detailed discussion will be given later.

\section{Thermodynamical significance of salinities of seawater and human blood and their relation with dissolved oxygen}

The diagram of the energy distribution in aqueous sodium chloride solution may be considered from two aspects, that of solute and that of solvent. First, let us consider about the energy which is held by the solution as a whole. Figure 10 shows the scheme of the thermodynamical energy of the solution as a function of the concentration of salt. With respect to the solutions of concentration lower than ca. $0.1 \mathrm{~mol} \mathrm{l}^{-1}$ of $\mathrm{NaCl}$, as shown in the previous discussions, the energy of the solution may be taken as dominated by the hydrogen bonding energy of the water molecules. It is assumed to be minus by setting the free water molecule as zero. The magnitude of the latter minus value may

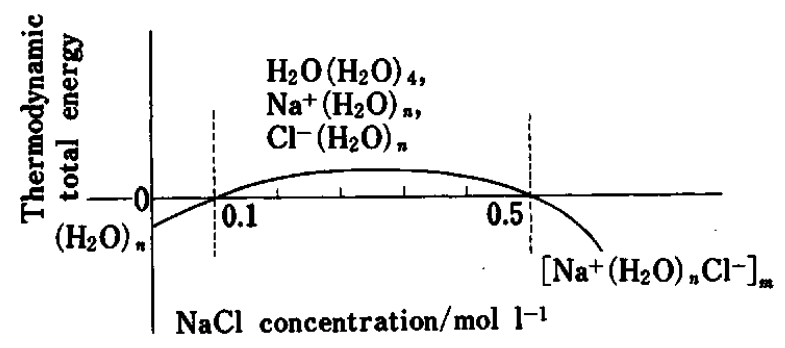

Fig. 10 Schematic diagram of energies of aqueous solutions of sodium chloride. be taken as equal to a few $\mathrm{kcal} \mathrm{mol}^{-1}$. By the introduction of the ions, the whole system of the solution holds the hydration energy of ions, which is minus, and the energy of dispersion entropy of the ions and that of the clusters of waters, which are plus. However, the energy of the whole system could be taken as minus as a whole. With the increase of the concentration, the energy of the dispersion entropy is increased, and the inner energy of the hydrogen bonding is decreased. It may be natural to assume that both cross at the concentration of $0.1 \mathrm{~mol} \mathrm{l}^{-1}$ of $\mathrm{NaCl}$, where the minus of the inner energy of the hydrogen bonding is balanced with the plus energy of the dispersion entropy. The bulk energy of the solutions with concentrations between 0.1 and $0.5 \mathrm{moll}^{-1}$ of $\mathrm{NaCl}$ is kept positive as can be seen in the data of the heats of infinite dilution. In the solutions of concentrations higher than ca. $0.5 \mathrm{~mol} \mathrm{l}^{-1}$, the bulk energy of the system can be taken as dominated by the interaction energy of the hydrated ions with minus values.

Besides the fluctuation of the refractive index, other anomalies have been noticed in the concentration range around $0.1 \mathrm{~mol} \mathrm{l}^{-1}$ of $\mathrm{NaCl}$ recently ${ }^{13}$; one, with respect to the spin-spin relaxation time, $T_{2}$, of the proton NMR, which refers to the minimal trend of $T_{2}$ at that specific concentration, and another, the observation of the latent heat of melting of the solution of the same concentration. ${ }^{14}$ The latter finding comes due to differential scanning calorimetry, DSC, on the frozen solution. The DSC measurements have revealed the presence of the cryohydrate crystal in the solutions of low concentrations. The literature ${ }^{15}$ shows that the cryohydrate crystal is formed only in solutions of very high concentrations, for example in a solution of $23.2 \%$ $\mathrm{NaCl}$ which presents an eutectic point of $-21.2^{\circ} \mathrm{C}$. However, recent measurements on DSC have revealed the formation of that crystal in the solutions of the concentration as low as $0.1 \mathrm{~mol} \mathrm{l}^{-1}$ of $\mathrm{NaCl}$. Figure 11 shows an example of the measurements. In practice, $0.1 \mathrm{mg}$ of the sample solution is weighed accurately and cooled down to $-60^{\circ} \mathrm{C}$ in the calorimeter. It is then heated at a rate of $1^{\circ} \mathrm{C} / \mathrm{min}$. Besides the strong signal at $0^{\circ} \mathrm{C}$ which refers to the melting of the ice, a small peak is noticed at around $-23^{\circ} \mathrm{C}$, which is close to the eutectic point of the cryohydrate crystal as cited above. It is natural to assign the small peak of DSC to the cryohydrate crystal. Then, the point of the finding is the fact that crystals are formed in some solutions of very low concentrations, which, in turn calls for care if the compound of the crystal is present in the solutional state of liquid. The present author assumes this compound exists. The reason for the latter statement is as follows. The earlier results of the quantum chemical calculation on the hydration energies on ions have shown some data about the sodium ion: that the binding energies per water molecule of the first (E-I), second (E-II), and third (E-III) hydration shells, for tetrahedral (Td) and octahedral (Oh) configurations are 


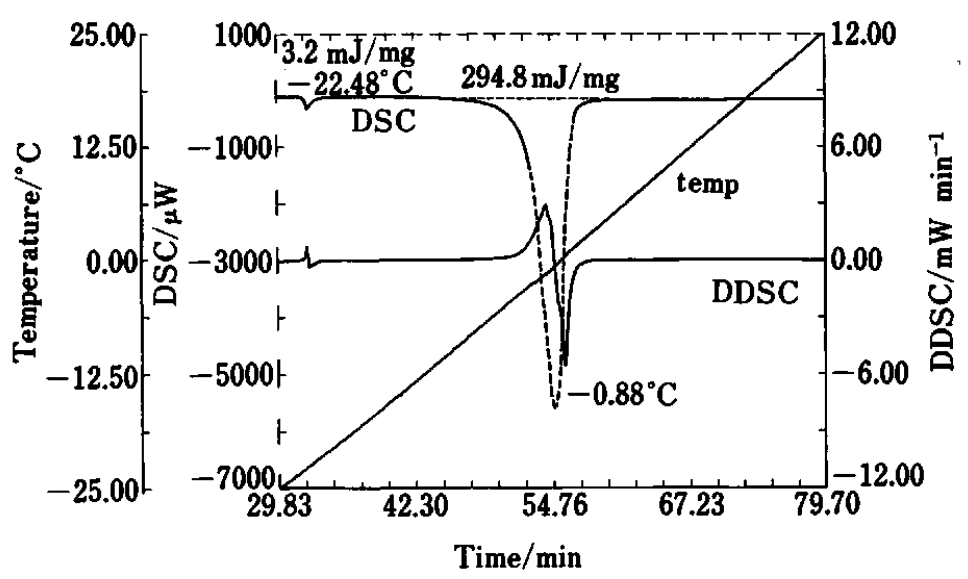

Fig. 11 Record of DSC of $0.1 \mathrm{~mol} \mathrm{l}^{-1} \mathrm{NaCl}$ solution.

E-I, $17.3(\mathrm{Td})$ and $15.4(\mathrm{Oh})$, E-II, $7.6(\mathrm{Td}, \mathrm{Oh})$, and E-III, 1.5 (Td, Oh). ${ }^{16}$ The data cited above suggest that the water molecules in the first and the second shells will be kept bound to the ion even in the solutional state of liquid, since they resist the kinetic force to remove the water molecules from the hydration shell. The third shell water molecules are assumed to merge freely with the bulk waters in the media. A situation such as that described here has called for the further assumption that the ions in the media of the solution may hold a maximum volume in the space of the solution. ${ }^{17}$ The latter volume has been named as the molecular space. The concept of the molecular space is due to the result of the measurement on the critical concentration of the cupric ions ${ }^{2}$ of $0.1 \mathrm{moll}^{-1}$ of $\mathrm{Cu}^{2+}$. The molecular space has been calculated by dividing the whole volume of 11 by the number of ions of $0.1 \mathrm{~mol}$ as a cube of $(20 \pm 5 \AA) .^{3}$ The quantum chemical calculation cited above has given the volumes of the hydrated ions (including hydration layers) as 681, 3352, 10730 (in hartrees) for the 1st, 2nd and 3rd shells, respectively. The latter values are slightly smaller than a cube of $(20 \pm 5 \AA){ }^{3} \quad$ For the evaluation of the experiments, other investigations have been made in the following way. By irradiation of the aqueous solution of polyvinylalcohol, PVA, by gamma-rays, it is possible to prepare a gel of PVA with a cross-linkage of some preferred value. ${ }^{17,18}$ By the use of this technique, PVA gels have been produced which are formed of cells of different spacings, ranging from 10 or 20 to $100 \AA$. The cupric ions ${ }^{17,18}$ or the spin-label ${ }^{19}$ compounds are embedded in the gels with the cell size of the molecular space. The ESR patterns of the embedded ions/molecules are good enough to refer to the critical size of the cell size, which allows the free motion of the ions/molecules. The critical size of the cell, roughly in the range around $(15 \AA)$ at the average, which has been determined experimentally, has assumed the presence of the critical volume for the free motion of ions/molecules.

\section{Significance of the salinities of seawater and human blood in nature: conclusive remark}

The anomalous fluctuations of the refractive indices in the solutions of 0.1 and $0.5 \mathrm{~mol} \mathrm{I}^{-1}$ of $\mathrm{NaCl}$ and of $\mathrm{D}_{2} \mathrm{O} / \mathrm{H}_{2} \mathrm{O}(20: 80 \mathrm{v} / \mathrm{v})$ mixtures become unobservable when oxygen is removed. If the aforementioned interpretation that those solutions of specific concentrations are the interfaces of two phases, one dominated by the entropy energy and the other by the inner energy, can be the case, oxygen is assumed to play a fundamental role in the work of the interface. The view of the present article on this is to assume that the anomalous fluctuation of the refractive index refers to the enhancement of the thermal agitation for the solution and that the latter agitation can be brought about by the oxygen. An interpretation for the latter statement follows.

Suppose each of the two phases cited above independently interacts with the outside energetically, i.e., taking in or out energy from outside. Each phase behaves independently from the other. Hence, those two phases can be taken as not being in equilibrium. Then, energy flow is assumed not to occur between those two phases, one dominated by entropy and the other by the inner energy. In this case, the thermal noise levels of both phases are assumed to be at the same low level.

This is the case when oxygen is absent. When oxygen is present, it is assumed to work as a catalyzer to bridge those two phases, actually to react with the water molecules in both phases leveling off the energy difference between the two phases. By the result of the latter reaction the solution of the specific concentration of the interface will yield enhanced thermal agitation. As to the real scheme of the work of the dissolved oxygen, further investigation will be necessary and can not be discussed in more detail. Only the work of oxygen in nature, particularly in the ocean and in the human blood, may be referred to. 


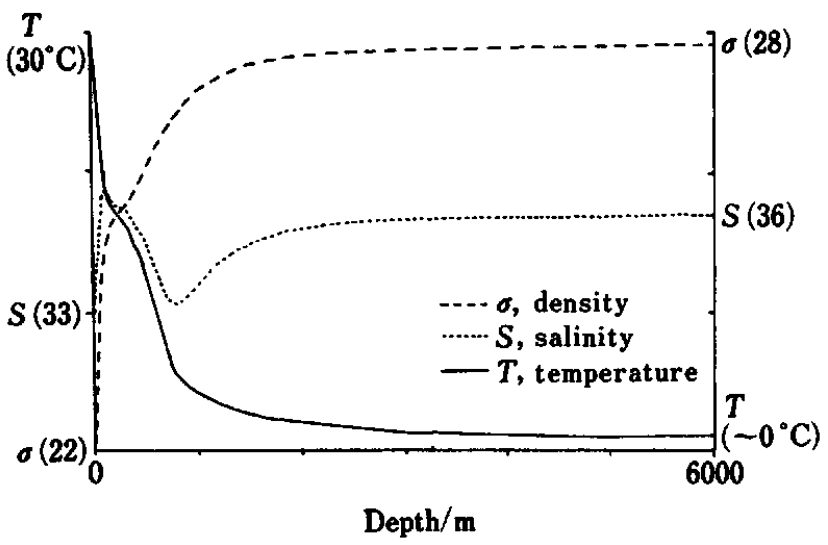

Fig. 12 Zonal structure of the sea as measured by Hakuhomaru of the Univ. of Tokyo, courtesy of Prof. Horibe, where $\sigma$, density, $S$, salinity and $T$, temperature are referred to by --, $\cdots$ and - , respectively, where $\sigma=[1-d$ (density) $\times 1000$.

Figure 12 is the sectional profile of the ocean, where the abscissa refers to the depth of the sea and the ordinate to the experimental measurables of the temperature, the density and the salinity. It is obvious from the figure that the ocean has a zonal structure: the surface zone, which is from the surface to the depth of around $2000 \mathrm{~m}$, and the lower zone, from ca. $2000 \mathrm{~m}$ to the bottom. In the surface zone, all measurables change violently, whereas, they are almost constant in the lower zone.

As cited above, the seawater holds the salinity of the interface. Therefore the origin of the zonal structure of the sea, i.e., the presence of the interface of two zones at around $2000 \mathrm{~m}$ must be due to another causes. The dissolved oxygen and the hydrostatic pressure are assumed to be the latter causes. The violent changes of the experimental measurables in the surface zone are due to the interaction of the sea with the atmosphere which holds the sunny energy. The surface zone seawater holds more oxygen: $4.7 \mathrm{ml} \mathrm{l}^{-1}$ of $\mathrm{O}_{2}$ than the lower one: $0.7 \mathrm{mll}^{-1}$ of $\mathrm{O}_{2}$ at $700 \mathrm{~m}$ depth. ${ }^{20}$ Accordingly, an interface exists with respect to the concentration of the oxygen at $700 \mathrm{~m}$ depth which divides the sea into two zones, one rich in oxygen and the other poor. Actually, oxygen shows a minimal value at the $700 \mathrm{~m}$ depth sea. Because the salinity of the seawater refers to the critical concentration, the surface water which holds oxygen can access thermal agitation from outside, whereas the lower zone water is assumed not to be able to access thermal agitation, as expected with the analogy for the refractive index measurements.

The real scheme of the energetics of the seawater must be complex and the effects of other elements such as that of the hydrostatic pressure must be taken into consideration.

A seawater model saline water with a concentration of $0.6 \mathrm{~mol} \mathrm{l}^{-1}$ of $\mathrm{NaCl}$ has been placed under up to

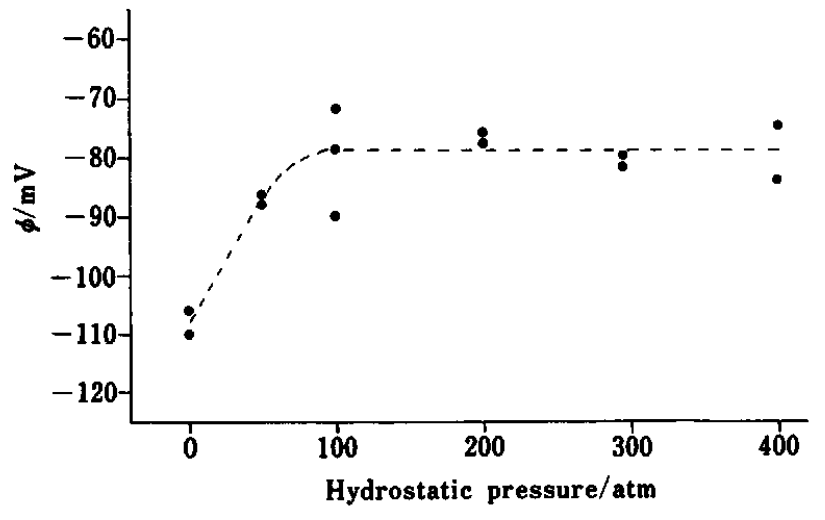

Fig. 13 Pressure dependence of $\phi$, the electromotive force of $0.60 \mathrm{~mol} \mathrm{l}^{-1} \mathrm{NaCl}$ solution.

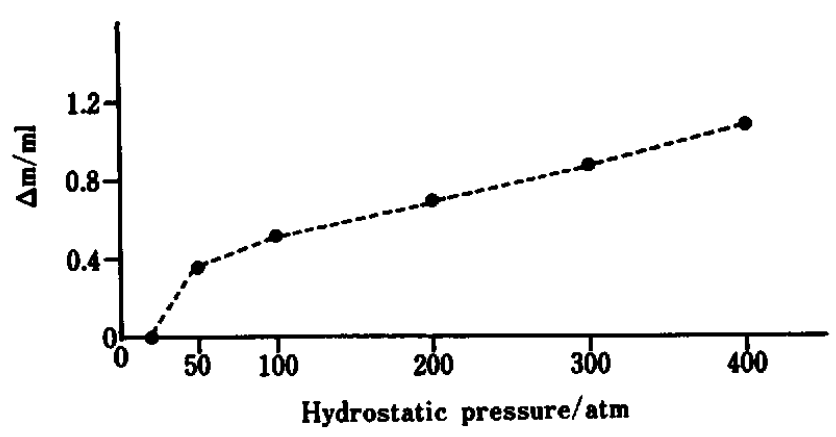

Fig. 14 Pressure dependence of volume contration, $\Delta m$.

$400 \mathrm{~atm}$ pressure, and the volume compression due to the pressure, $-\Delta m$, and the change of the electromotive force, $\phi$, have been measured. ${ }^{21}$ Both $\Delta m$ and $\phi$ have shown drastic changes in the range from zero to $100 \mathrm{~atm}$; over $100 \mathrm{~atm}$, the change for $\phi$ is zero or small for $\Delta m$ (Figs. 13 and 14). These results suggest that the condition of the water molecules are changed discontinually at around 100 atms, from a porous structure to a tight one. The basis of the zonal structure of the sea may first lie on the discontinual contraction of the volume. Figure 15 shows the $\phi$ values observed in $0.60 \mathrm{moll}^{-1} \mathrm{NaCl}$ solution at the normal pressure with the silver plate electrodes of different areas, $A$ 's. The effect of deaeration is obvious in the figure. The $\phi$ values in the deaerated solution, open circles, do not show the dependence on $A$ values, whereas those in the solutions which contain dissolved oxygen, solid circles, show a marked dependence on $A$ of relatively small value. As the readings of $\phi$ are compared to each other for the $\mathrm{NaCl}$ solutions of different concentrations, those for the solutions of 0.5 or $0.6 \mathrm{~mol} \mathrm{l}^{-1}$ have been found unstable, i.e., fluctuate. The point of the results of the measurements lies in the fact that the $A$ dependence of $\phi$ suggests two different electrochemical behaviors, one dependent on $A$ and the other not. As a matter of the electrochemistry, $\phi$ of $A$ dependence is a peculiar phenomenon and needs a 


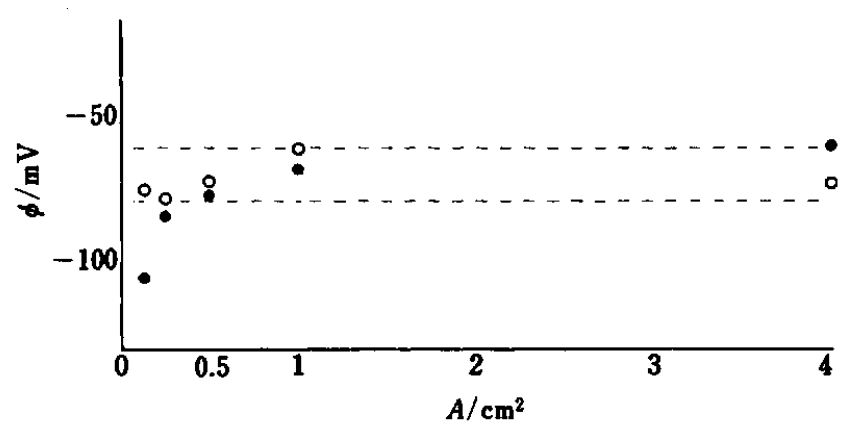

Fig. 15 Effect of deaeration on the electromotive force of $0.60 \mathrm{~mol} \mathrm{l}^{-1} \mathrm{NaCl}$. A: surface area of the electrode.

more detailed investigation in the future. The facts cited above at least suggest that the violent changes of the measurables in the surface waters of the sea are strongly related with the volume contraction as well as with the concentration of oxygen. Here a speculative scheme can be presented with respect to the energy flow of the sea.

Suppose some locally unequal salinities are produced in the surface zone water, this would imply the dilution of the seawater by the running in of the riverwater or its thickening by the evaporation. The latter events refer either to the increase of the entropy energy or the inner energy. If oxygen is present there, the energies of the waters of different salinities of entropy or of inner energy will be able to flow in and out freely among the sites of different salinities, which can be expected from the results of the measurements of the fluctuation of the refractive index. This is the case of the surface water zone. With respect to the lower zone, the oxygen content is low, almost negligible. Hence, energy flow can not occur amongst the sites of different salinities, even if those sites occur. Thus the constant values of the measurables of the lower zone seawater happen.

Oxygen molecules may also work similarly in the human blood. As mentioned before, the salinity of the human blood, $0.1 \mathrm{moll}^{-1}$ of $\mathrm{NaCl}$, is a critical concentration which divides two phases of water, one the hydrogen bond network and the other the assembly of the clusters of water molecules. Fluctuations of the refractive index are enhanced at this specific concentration only under the presence of oxygen. The anomalous fluctuation observed may be attributed to an active response to the thermal interaction with outside. The artery blood which contains oxygen will be able to access energy flow from outside, whereas the vein blood which has no/almost no oxygen will not.

The salinities of the human blood and the seawater refer to thermodynamically important concentrations. Oxygen plays an important role for the holders, the human being and the earth. The oxygen effect has a more fundamental relation. As a matter of analytical science, I note that the measurement of the fluctuation of the refractive index, which has not been taken up much before, has brought to light such an important finding as the anomalous fluctuation in the solutions of specific concentrations.

\section{References}

1. S. Fujiwara and Y. Nishimoto, Spectrosc. Lett., 17, 633 (1984).

2. S. Fujiwara and S. Hayashi, J. Chem. Phys., 43, 23 (1965).

3. V. B. Parker, NBS Technical Report (1965). S. Fujiwara, Geochem. J., 13, 225 (1979).

4. Various reports have been made at the 1st Eurasia Conference on Chemistry of Solution (1988), Bangkok: X-ray studies by H. Ohtaki (Japan) and I. Persson (Sweden), Computer simulation by P. Bopp (FRG) and H. Kleeberg and P. Luck (FRG), EXAFS by S. Ahrland et al. (Sweden), Raman by D. E. Irish et al. (Canada), and by G. Goege (FRG), Moessbauer by K. G. Belyaeva (USSR), Dielectic and ultrasonic study by R. Ptowiec (Poland), NMR by P. Kreishman (USA), by Li Zhifen $e t$ al. (PRC) and by W. Jinsart (Thailand), IR by SI-Joong Kim (Korea).

5. For example: H. Ohtaki, Rev. Inorg. Chem., 4, 103 (1982).

6. J. P. Limtrakul, S. Fujiwara and B. M. Rode, Anal. Sci., 1, 29 (1985).

7. S. Fujiwara and Y. Nishimoto, Proc. Japan Academy, 59, B333 (1983).

8. S. Fujiwara, Y. Nishimoto and F. Arakawa, Anal. Sci., 1, 23 (1985).

9. S. Fujiwara, F. Arakawa and O. Toi, Anal. Sci, 3, 213 (1987).

10. G. C. Lie and E. Clementi, IBM Technical Report, KGN-36 (1985).

11. K. Heinzinger, The 1st Eurasia Conference on Chemistry of Solution (1988), Bangkok.

12. F. Franks, "Water", Vol. 1, p. 172, Plenum Press, New York, London (1972).

13. Private communication of Prof. T. Watanabe of the University of Fisheries.

14. Private communication of Miss Y. Nishimoto of DainiSeiko Corp.

15. Gmelins Handbuch der Anorganischen Chemie, 8 Auf. System Nr. 21, p. 332 (1928) and Neu Bearbeitete Ergaenzungsband System Nr. 21, p. 4 (1973), Verlag Chemie.

16. S. Fujiwara and B. M. Rode, Bull. Chem. Soc. Jpn., 52, 3221 (1979).

17. S. Fujiwara, K. Nagashima, H. Morita and Y. Kanaoka, Bull. Chem. Soc. Jpn., 50, 2851 (1977).

18. S. Fujiwara, S. Katsumata and T. Seki, J. Phys. Chem., 71, 115 (1967).

19. T. Watanabe, T. Yahagi and S. Fujiwara, J. Am. Chem. Soc., 102, 5187 (1980).

20. Report of CYGNUS and CEPHEUS expedition of the Institute of Oceanography of the University of Tokyo.

21. S. Fujiwara and A. Takatsu, Anal. Sci., 1, 231 (1985).

(Received February 1, 1988)

(Accepted February 29, 1988) 